\title{
ON THE VALUE DISTRIBUTION OF QUASIMEROMORPHIC MAPS
}

\author{
SEPPO RICKMAN
}

\section{Introduction}

Let $G$ be a domain in the euclidean $n$-space $R^{n}$ and let $n \geq 2$. A continuous map $f: G \rightarrow R^{n}$ is called quasiregular if $f$ is $\mathrm{ACL}^{n}$, i.e. $f$ is absolutely continuous on lines with $L_{\text {loc }}^{n}$ integrable partial derivatives, and if

$$
\left|f^{\prime}(x)\right|^{n} \leq K J(x, f)
$$

holds a.e. in $G$ for some $K, 1 \leq K<\infty$. Here $f^{\prime}(x)$ is the formal derivative of $f$ at $x,\left|f^{\prime}(x)\right|$ its supnorm, and $J(x, f)$ the jacobian determinant of $f$ at $x$. A continuous map $f: G \rightarrow \bar{R}^{n}=R^{n} \cup\{\infty\}$, where now $G$ is a domain in the compactified space $\bar{R}^{n}$, is defined to be quasimeromorphic by means of the definition of quasiregularity and auxiliary Möbius transformations (see [5]). The smallest constant $K \geq 1$ in (1.1) is called the outer dilatation $K_{O}(f)$ of $f$ and the smallest constant $K \geq 1$ for which

$$
J(x, f) \leq K \inf _{|h|=1}\left|f^{\prime}(x) h\right|^{n}
$$

holds a.e. in $G$ is called the inner dilatation $K_{I}(f)$ of $f \cdot K(f)=$ $\max \left(K_{O}(f), K_{I}(f)\right)$ is the maximal dilatation of $f$.

Quasiregular and quasimeromorphic maps have in recent years turned out to form a natural real $n$-dimensional generalization of the analytic and meromorphic functions in the plane. For the existing main features of the theory we refer to $[4 ; 5 ; 6]$.

No systematic study has been made of value distribution theory of quasimeromorphic maps in dimensions $n \geq 3$ earlier. However, some treated problems deserve mentioning. It follows from [5, Theorem 4.4] that a quasimeromorphic map $f: R^{n} \rightarrow \bar{R}^{n}$ which omits a set of positive 
conformal capacity must be constant. One of the main open problems is whether a Picard's theorem is true, i.e. whether a quasimeromorphic map $f: R^{n} \rightarrow \bar{R}^{n}$ omitting three points (or maybe a number depending on the dimension) must be constant. On the other hand, it was proved in [9] for dimension $n=3$ that if each point $a$ in a countable set $A \subset \bar{R}^{n}$ is attached a number $\delta(a)$ such that $0<\delta(a) \leq 1$ and $\sum_{a} \delta(a) \leq 2$, then a quasimeromorphic map $f: R^{n} \rightarrow \bar{R}^{n}$ can be constructed which has defect $\delta(a)$ in the point $a$ and no other defects, more precicely, $f$ is given so that (see Section 2 for notation)

$$
\begin{array}{ll}
\lim _{r \rightarrow \infty} \frac{n(r, a)}{A(r)}=1-\delta(a) & \text { if } a \in A, \\
\lim _{r \rightarrow \infty} \frac{n(r, a)}{A(r)}=1 & \text { if } a \in \bar{R}^{n} \backslash A .
\end{array}
$$

The given construction modified to the case $n=2$ gives a rather simple solution for a restricted inverse problem of Nevanlinna's second main theorem for plane quasimeromorphic maps. Such a theorem is given in [2, Satz 17] where e.g. the defect $\delta(a)$ at $a$ is defined as

$$
\delta(a)=\liminf _{r \rightarrow \infty}\left(1-\frac{N(r, a)}{T(r)}\right) .
$$

We point out that "restricted" above actually means more than ignoring the indices of multiplicity because a defect defined by (1.2) can be negative for a quasimeromorphic map.

In this paper we shall consider various questions of value distribution of quasimeromorphic maps $f: R^{n} \rightarrow \bar{R}^{n}$ with respect to an exhaustion by balls $B(r)=\left\{x \in R^{n}|| x \mid<r\right\}$. Almost all results can be considered as to present a balance between some value distribution quantities taken with respect to balls with two different radii. We work all over with "nonintegrated" concepts, i.e. we do not integrate with respect to $r$, and we do not know if such an integration could give more information as it does in the Nevanlinna theory.

After preliminaries in Section 2 we start in Section 3 by showing that a minimum-maximum balance for the counting function $n(r, a)$ always holds except possibly for a set of capacity zero. Here we also introduce in a simple form the main tool used in the proofs based on inequalities for moduli of path families.

The results of Section 4 serve on one hand as examples of relating averages of value distribution over submanifolds. For simplicity we consider only the whole space and $(n-1)$-dimensional spheres but it is possible to 
extend the results also to much more general sets. Leaving out an exceptional set of the radii $r$ we obtain results well known for $n=2$ from the theory of covering surfaces by Ahlfors. On the other hand, the theorems of Section 4 are effectively used in Section 5 .

As to an analog for Nevanlinna's first main theorem one could hope that an inequality of the type $\lim \sup _{r \rightarrow \infty} N(r, a) / T(r) \leq M$ is true where $N(r, a)$ and $T(r)$ are suitable integrals of $n(t, a)$ and $A(t)$ respectively and where $M$ depends only on the dimension $n$ and the dilatation $K(f)$. Such a result is not even known for $n=2$. However, it is possible to obtain in all dimensions relations in this direction on the "nonintegrated level" which also in a qualitative sense are best possible. This will be shown in Section 5 where also a more detailed discussion is given.

Many of the results of this paper, especially those of Section 5, seem to be new also for meromorphic functions in plane. I want to thank Sakari Toppila for constructing simultaneously examples of meromorphic functions in [10] which in a nice way complete the results of Section 5.

\section{Preliminary results}

2.1. Notation and terminology. We shall mainly use the terminology in [4]. For basic properties of quasimeromorphic maps we refer to $[4,5]$. For $x \in R^{n}$ and $r>0$ we let $B(x, r)$ denote the ball $\left\{y \in R^{n}|| x-y \mid<r\right\}$ and $S(x, r)$ the $(n-1)$-dimensional sphere $\left\{y \in R^{n}|| x-y \mid=r\right\}$. We also use the following abbreviations:

$$
B(r)=B(0, r), \quad S(r)=S(0, r), \quad S=S(1) .
$$

The Lebesgue measure in $R^{n}$ is denoted by $m$ and the normalized $k$ dimensional $(k \leq n)$ Hausdorff measure by $\mathscr{H}^{k}$. For the $(n-1)$ dimensional measure $\mathscr{H}^{n-1}(S)$ of the unit sphere we use the notation $\omega_{n-1}$. The spherical chordal metric in $\bar{R}^{n}$ is denoted by $q$.

Since a nonconstant quasimeromorphic map is always discrete, open, and sensepreserving, a local index $i(x, f) \geq 1$ is defined in the domain of definition (see [4]). If $f: R^{n} \rightarrow \bar{R}^{n}$ is a nonconstant quasimeromorphic map, we use the local index to define for $r>0$ and $a \in \bar{R}^{n}$ the counting function:

$$
n(r, a)=\sum_{x \in f^{-1}(a) \cap \overline{B(r)}} i(x, f) .
$$

With this definition $n(r, a)$ is the number of points of the preimage of $a$ in the ball $\bar{B}(r)$ with multiplicity regarded. 
If $E \subset R^{n}$ is $\mathscr{H}^{k}$-measurable, we denote by $\sigma^{k}(\mathrm{E})$ the $k$-dimensional spherical measure, i.e.

$$
\sigma^{k}(E)=\int_{E} \frac{1}{\left(1+|y|^{2}\right)^{k}} d \mathscr{H}^{k}(y) .
$$

The function $y \mapsto n(r, y)$ is upper semicontinuous and we can define the average of the distribution of values taken in the ball $\bar{B}(r)$ over the whole space $\bar{R}^{n}$ with respect to the spherical metric, called spherical average, as

$$
A(r)=\frac{1}{\lambda_{n}} \int_{R^{n}} \frac{n(r, y)}{\left(1+|y|^{2}\right)^{n}} d m(y)
$$

where $\lambda_{n}=\sigma^{n}\left(R^{n}\right)$ is the total spherical measure of $\bar{R}^{n}$. We shall also need averages over $(n-1)$-spheres in $\bar{R}^{n}$, therefore let $Y$ be a sphere $\left\{y \in \bar{R}^{n} \mid q(y, x)=s\right\}$. We denote the corresponding average over $Y$ by $v(r, Y)$, i.e.

$$
v(r, Y)=\frac{1}{\sigma^{n-1}(Y)} \int_{Y \cap R^{n}} \frac{n(r, y)}{\left(1+|y|^{2}\right)^{n-1}} d \mathscr{H}^{n-1}(y) .
$$

For the special case $Y=S(t)$ we also denote $v(r, t)=v(r, S(t))$. For this case we have

$$
v(r, t)=\frac{1}{\omega_{n-1}} \int_{S} n(r, t y) d \mathscr{H}^{n-1}(y) .
$$

2.2. Modulus of path families and quasimeromorphic maps. All our proofs depend essentially on inequalities of moduli of path families. If $I$ is a family of nonconstant paths in $\bar{R}^{n}$, we define $F(T)$ to be the set of all nonnegative extended real valued Borel functions of $R^{n}$ such that for every locally rectifiable $\gamma \in \Gamma$ we have

$$
\int_{\gamma} \varrho d s \geq 1
$$

The modulus of $\Gamma$ is then defined as

$$
M(\Gamma)=\inf _{\varrho \in F(T)} \int_{R^{n}} \varrho^{n} d m .
$$

For discussions of inequalities for moduli of path families in connection with quasiregular maps we refer to [4, pp. 15-17], [7], and [12]. 
The basic tool for us will be the inequality in Lemma 2.3 combined with a result on maximal path lifting stated in Lemma 2.4. We need the following terminology for path lifting. Let $f: G \rightarrow \bar{R}^{n}$ be a continuous, discrete, and open map of a domain $G$ and let $\beta:[a, b] \rightarrow \bar{R}^{n}$ be a path. Suppose $x \in f^{-1}(\beta(a))$. We call a path $\alpha: J \rightarrow G$ a partial $f$-lifting of $\beta$ starting at $x$ if $J$ is an interval such that $a \in J \subset[a, b], \alpha(a)=x$, and $f \circ \alpha=$ $\beta \mid J$. A maximal $f$-lifting of $\beta$ starting at $x$ is a partial $f$-lifting $\alpha: J \rightarrow G$ starting at $x$ such that there exists no partial $f$-lifting $\alpha^{\prime}: J^{\prime} \rightarrow G$ with $J \subset J^{\prime}, J \neq J^{\prime}$ and $\alpha^{\prime} \mid J=\alpha$. With slight modifications Lemma 2.3 follows from Theorem 3.1 in [12] by letting $E_{0}$ to be the branch set $B_{f}$ (cf. $[4,8.3]$ ) and Lemma 2.4 is Theorem 1 in [8].

2.3. L e m ma. Let $f: G \rightarrow \bar{R}^{n}$ be a nonconstant quasimeromorphic map, $\Gamma^{*}$ a family of paths in $G, \Gamma$ a family of paths $\beta:[a, b] \rightarrow \bar{R}^{n}$, and let $m$ be a positive integer. Suppose that every $\beta \in \Gamma$ has partial $f$ liftings $\alpha_{1}, \ldots, \alpha_{m}$ in $\Gamma^{*}$ starting at points in $f^{-1}(\beta(\alpha))$ such that

$$
\text { card }\left\{j \mid \alpha_{j}(t)=x\right\} \leq i(x, f) \text { for all } x \in G \text { and } t \in[a, b] \text {. }
$$

Then

$$
M(\Gamma) \leq \frac{K_{I}(f)}{m} M\left(\Gamma^{*}\right)
$$

2.4. L e m m a. Let $f: G \rightarrow \bar{R}^{n}$ be continuous, discrete, and open. Let $\beta:[a, b] \rightarrow \bar{R}^{n}$ be a path, let $x_{1}, \ldots, x_{k}$ be points in $f^{-1}(\beta(a))$, and set

$$
p=\sum_{i=1}^{k} i\left(x_{i}, f\right) .
$$

Then there are maximal f-liftings $\alpha_{1}, \ldots, \alpha_{p}$ of $\beta$ starting at $x_{1}, \ldots, x_{k}$ such that

(1) $\operatorname{card}\left\{j \mid \alpha_{j}(a)=x_{i}\right\}=i\left(x_{i}, f\right)$ for $1 \leq i \leq k$,

(2) $\operatorname{card}\left\{j \mid \alpha_{j}(t)=x\right\} \leq i(x, f) \quad$ for all $x \in G$ and $t \in[a, b]$.

\section{Balance with exceptional set of capacity zero}

In this section we shall give in a simple form a principle which turns out to be fruitful in studying the balance of value distribution between different sets. The result of this section (Theorem 3.2) can be called a minimum-maximum balance for values taken in balls with different radii. 
The concept of a set of capacity zero is defined in $[5,2.12]$. We need the following simple lemma.

3.1. L e m m a. Let $f: R^{n} \rightarrow \bar{R}^{n}$ be a quasimeromorphic map with $\infty$ as an essential singularity. Then there exists a set $E$ of capacity zero such that for all $z \in \bar{R}^{n} \backslash E$

$$
\lim _{r \rightarrow \infty} n(r, z)=\infty \text {. }
$$

Proof. By [5, 4.4] eap $\mathbf{C} f\left(R^{n} \backslash B(r)\right)=0$ for all $r>0$. Then the set

$$
\mathbb{E}=\underset{r>0}{\cup} \mathbf{C} f\left(R^{n} \backslash B(r)\right)=\bigcup_{k=1}^{\infty} \mathbf{C} f\left(R^{n} \backslash B(k)\right)
$$

is of zero capacity. If $z \in \bar{R}^{n} \backslash E$, there exists a sequence $\left(x_{i}\right)$ of points in $R^{n}$ such that $x_{i} \neq x_{j}$ if $i \neq j$ and $f\left(x_{i}\right)=z$ for all $i$, hence $n(r, z) \rightarrow \infty$ as $r \rightarrow \infty$.

3.2. Th e o r e m. Let $f: R^{n} \rightarrow \bar{R}^{n}$ be a nonconstant quasimeromorphic map, let $A$ and $B$ be disjoint sets in $\bar{R}^{n}$, let $B$ have positive capacity, and let $\theta>1$. If

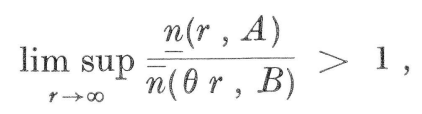

where

$$
\begin{aligned}
& \underline{n}(r, A)=\inf _{y \in A} n(r, y), \\
& \bar{n}(\theta r, B)=\sup _{z \in B} n(\theta r, z),
\end{aligned}
$$

then $A$ is of zero capacity.

Proof. We denote the left hand side of (3.3) by $c$. Suppose first that $f$ has an extension to a quasimeromorphic map $f^{*}: \bar{R}^{n} \rightarrow \bar{R}^{n}$. Then for $y \neq f^{*}(\infty) n(r, y)$ is a constant $k$ for large $r$ and $n(r, z) \leq k$ for all $z \in \bar{R}^{n}$ and $r>0$. Hence $c \leq 1$. We may therefore assume that $\infty$ is an essential singularity of $f$. By Lemma $3.1 \bar{n}(\theta r, B) \rightarrow \infty$ as $r \rightarrow \infty$. We choose a sequence $r_{1}<r_{2}<\ldots \rightarrow \infty$ with

$$
\lim _{i \rightarrow \infty} \frac{n\left(r_{i}, A\right)}{\bar{n}\left(\theta r_{i}, B\right)}=c .
$$

Let $\Gamma$ be the family of paths $\gamma:[0,1] \rightarrow \bar{R}^{n}$ with $\gamma(0) \in A, \gamma(1) \in B$. Fix $i$, let $\gamma \in \Gamma$ and denote $y=\gamma(0)$. If $\left\{x_{1}, \ldots, x_{k}\right\}=f^{-1}(y) \cap \bar{B}\left(r_{i}\right)$, then 


$$
p=\sum_{j=1}^{k} i\left(x_{j}, f\right) \geq \underline{n}\left(r_{i}, A\right) .
$$

Let $\alpha_{1}, \ldots, \alpha_{p}$ be the maximal $f \mid B\left(\theta r_{i}\right)$-liftings starting at the points $x_{1}, \ldots, x_{k}$ given by Lemma 2.4. At most $\bar{n}\left(\theta r_{i}, B\right)$ of these paths may end in $B\left(\theta r_{i}\right)$, hence at least $p-\bar{n}\left(\theta r_{i}, B\right) \geq \underline{n}\left(r_{i}, A\right)-$ $\bar{n}\left(\theta r_{i}, B\right)=m_{i}$ of the paths $\alpha_{1}, \ldots, \alpha_{p}$ have one endpoint in $\partial B\left(\theta r_{i}\right)$. Let $\Gamma_{\gamma}^{*}$ be the set of those paths. We set $\Gamma^{*}=\cup\left\{\Gamma_{\gamma}^{*} \mid \gamma \in \Gamma\right\}$. By Lemma 2.3

$$
M(\Gamma) \leq \frac{K_{I}(f)}{m_{i}} M\left(\Gamma^{*}\right) .
$$

On the other hand, by $[11,7.5]$

$$
M\left(\Gamma^{*}\right) \leq \omega_{n-1}(\log \theta)^{1-n} .
$$

If $c>1$, it follows that $m_{i} \rightarrow \infty$ as $i \rightarrow \infty$. Hence $M(\Gamma)=0$ by $(3.4)$ and (3.5). It follows by [13] that $A$ is of capacity zero. The theorem is proved.

3.6. R e m a r k. From the proof it follows that instead of a fixed $\theta$ one obtains the same conclusion if we let $\theta$ vary in (3.3) with $r$, call it $\theta_{\text {, }}$ such that

$$
\lim _{r \rightarrow \infty} \underline{n}(r, A)\left(\log \theta_{r}\right)^{n-1}=\infty
$$

\section{Averages of the counting function}

We shall next show how to obtain a balance between averages of the counting function over $(n-1)$-dimensional spheres. As a consequence we get also the relationship to the average $A(r)$. The results will be applied in the next section to prove connections between the counting function and the spherical average. This section serves as an example how it is possible to relate value distribution averages obtained by integration over submanifolds. One can use similar technics to obtain corresponding results also for more general sets.

The following result for concentric spheres will be basic for all later applications.

4.1. The orem. Let $\theta, c>1$, let $0<s, t<\infty$. and let $f: R^{n} \rightarrow \bar{R}^{n}$ be a nonconstant quasimeromorphic map. Then

$$
c v(\theta r, t) \geq v(r, s)-\frac{K_{I}(f)|\log (t / s)|^{n-1}}{(1-1 / c)(\log \theta)^{n-1}}
$$


for $r>0$. Furthermore, there exists $r_{0}>0$ such that for $r \geq r_{0}$

$$
c \nu(\theta r, t) \geq v(r, s) \text {. }
$$

Proof. We may assume $s<t$. Let $r>0$ and set

$$
E=\{y \in S \mid c n(\theta r, t y)<n(r, s y)\} \text {. }
$$

Then

$$
\begin{aligned}
& \int_{S} n(\theta r, t y) d \mathscr{H}^{n-1}(y) \geq \int_{S \backslash E} n(\theta r, t y) d \mathscr{H}^{n-1}(y) \\
& \geq \frac{1}{c} \int_{S \backslash E} n(r, s y) d \mathscr{H}^{n-1}(y) \\
& =\frac{1}{c}\left(\int_{S} n(r, s y) d \mathscr{H}^{n-1}(y)-\int_{E} n(r, s y) d \mathscr{H}^{n-1}(y)\right) .
\end{aligned}
$$

To estimate the last integral in (4.4) let

$$
E_{k}=\{y \in E \mid n(r, s y)=k\}, \quad k=1,2, \ldots .
$$

For $y \in S$ let $\beta_{y}:[s, t] \rightarrow R^{n}$ be the path defined by $\beta_{y}(u)=u y$. Set

$$
\Gamma_{k}=\left\{\beta_{y} \mid y \in E_{k}\right\} .
$$

Let $y \in E_{k}$ and let $\alpha_{1}, \ldots, \alpha_{k}$ be the maximal $f \mid B(\theta r)$-liftings of $\beta_{y}$ starting at the points of $f^{-1}(s y) \cap \bar{B}(r)$ given by Lemma 2.4. Since $y \in E$, at least $n(r, s y)-n(r, s y) / c=(1-1 / c) k$ of the lifts $\alpha_{1}, \ldots, \alpha_{k}$ must end in $\partial B(\theta r)$. Let the family of those lifts when $y$ runs over the set $E_{k}$ be $\Gamma_{k}^{*}$. Then by Lemma 2.3

$$
k(1-1 / c) M\left(\Gamma_{k}\right) \leq K_{I}(f) M\left(\Gamma_{k}^{*}\right) .
$$

On the other hand, $M\left(\Gamma^{*}\right)=\sum_{k} M\left(\Gamma_{k}^{*}\right)$ by $[11,6.7]$ where $\Gamma^{*}=\cup_{k} \Gamma_{k}^{*}$, and

$$
M\left(\Gamma_{k}\right)=\frac{\mathscr{H}^{n-1}\left(E_{k}\right)}{(\log (t / s))^{n-1}}
$$

by $[11,7.7]$. Hence

$$
\begin{aligned}
K_{I}(f) M\left(\Gamma^{*}\right) & \geq \frac{1-1 / c}{(\log (t / s))^{n-1}} \sum_{k} k \mathscr{H}^{n-1}\left(E_{k}\right) \\
& =\frac{1-1 / c}{(\log (t / s))^{n-1}} \int_{E} n(r, s y) d \mathscr{H}^{n-1}(y) .
\end{aligned}
$$

But $M\left(\Gamma^{*}\right) \leq \omega_{n-1}(\log \theta)^{1-n}$ and the final estimate is then 


$$
\int_{E} n(r, s y) d \mathscr{H}^{n-1}(y) \leq \frac{K_{I}(f) \omega_{n-1}(\log (t / s))^{n-1}}{(1-1 / c)(\log \theta)^{n-1}} .
$$

This with (4.4) gives (4.2).

To prove (4.3) we may assume that $\infty$ is an essential singularity for otherwise it is trivial. If $F_{k}(r)$ denotes the set of points $y \in S$ for which $n(r, s y) \geq k$, then by 3.1

$$
\begin{aligned}
\lim _{r \rightarrow \infty} v(r, s) & \geq \lim _{j \rightarrow \infty} \frac{1}{\omega_{n-1}} \int_{F_{k}(j)} k d \mathscr{H}^{n-1}=k \lim _{j \rightarrow \infty} \frac{\mathscr{H}^{n-1}\left(F_{k}(j)\right)}{\omega_{n-1}} \\
& \geq k \frac{\mathscr{H}^{n-1}(S \backslash H)}{\omega_{n-1}}
\end{aligned}
$$

where $H$ is of zero capacity, hence $\mathscr{H}^{n-1}(S \backslash H)=\omega_{n-1}$. We conclude $v(r, s) \rightarrow \infty$ as $r \rightarrow \infty$. Then (4.3) follows by applying (4.2) to a smaller $c$. The theorem is proved.

4.5. R e m a r k. From (4.2) one observes that we may let $\theta$ vary with $r$, call it $\theta_{r}$, such that

$$
\lim _{r \rightarrow \infty} v(r, s)\left(\log \theta_{r}\right)^{n-1}=\infty,
$$

and still obtain the conclusion $c v\left(\theta_{r}, r, t\right) \geq v(r, s)$ corresponding to (4.3). A similar observation can be made about the constant $c$, in particular, for all $\theta>1$

$$
\liminf _{r \rightarrow \infty} \frac{v(\theta r, t)}{v(r, s)} \geq 1
$$

4.6. $\mathrm{R}$ e $\mathrm{m}$ a $\mathrm{rk}$. Let $f: R^{n} \rightarrow R^{n}$ be quasiregular and set for $r>0$

$$
M(r)=\max _{|x|=r}|f(x)| .
$$

Then a simplified version of the proof of Theorem 4.1 gives

$$
(\log M(\theta r))^{n-1} \geq \frac{(\log \theta)^{n-1}}{K_{I}(f)} v(r, 1)
$$

whenever $r>0$ and $\theta>1$.

We next use Theorem 4.1 to draw a connection to the spherical average $A(r)$.

4.8. $\mathrm{Th}$ e o r e m. Let $\theta, c>1$, let $Y$ be an $(n-1)$-dimensional sphere, and let $f: R^{n} \rightarrow \bar{R}^{n}$ be a nonconstant quasimeromorphic map. Then 


$$
\begin{aligned}
& \frac{1}{c} v(r / \theta, Y)-\frac{K_{I}(f) \tau}{(c-1)(\log \theta)^{n-1}} \leq A(r) \\
& \leq c v(\theta r, Y)+\frac{K_{I}(f) \tau}{(1-1 / c)(\log \theta)^{n-1}}
\end{aligned}
$$

for $r>0$ where $\tau \in] 0, \infty[$ depends only on $n$ and the spherical diameter of $Y$. Furthermore, there exists $r_{0}>0$ such that

$$
\frac{1}{c} v(r / \theta, Y) \leq A(r) \leq c v(\theta r, Y)
$$

for $r>r_{0}$.

Proof. By performing a Möbius transformation which preserves spherical distances if necessary we may assume that $Y=S(t)$ for some $t>0$. By $(4.2)$

$$
c v(\theta r, t) \geq v(r, s)-d\left|\log \frac{t}{s}\right|^{n-1}
$$

for $0<s<\infty$ where we have denoted $d=K_{I}(f)(1-1 / c)^{-1}(\log \theta)^{1-n}$. We multiply (4.11) by $s^{n-1} /\left(1+s^{2}\right)^{n}$ and integrate with respect to $s$ :

$$
\begin{aligned}
& c v(\theta r, t) \int_{0}^{\infty} \frac{s^{n-1}}{\left(1+s^{2}\right)^{n}} d s \\
& \geq \int_{0}^{\infty} \frac{v(r, s) s^{n-1}}{\left(1+s^{2}\right)^{n}} d s-d \int_{0}^{\infty} \frac{s^{n-1}|\log (t / s)|^{n-1}}{\left(1+s^{2}\right)^{n}} d s .
\end{aligned}
$$

This gives

where

$$
c v(\theta r, t) \geq A(r)-d h(t)
$$

$$
h(t)=\frac{\int_{0}^{\infty} \frac{s^{n-1}|\log (t / s)|^{n-1}}{\left(1+s^{2}\right)^{n}} d s}{\int_{0}^{\infty} \frac{s^{n-1}}{\left(1+s^{2}\right)^{n}} d s}
$$

is finite and depends only on $n$ and the spherical diameter of $Y$. With $\tau=h(t)$ gives the right hand side inequality in (4.9). The left hand side inequality is proved similarly by integrating the inequality

$$
c v(r, s) \geq v(r / \theta, t)-d\left|\log \frac{t}{s}\right|^{n-1}
$$


with respect to $s$ after multiplication again by $s^{n-1} /\left(1+s^{2}\right)^{n}$. The last statement is proved as in the proof of (4.3).

4.12. $\mathrm{R}$ e $\mathrm{m}$ a r k. A remark corresponding to 4.5 can be made also to Theorem 4.8.

4.13. Corollary. Let $\theta, c>1$, let $Y_{1}$ and $Y_{2}$ be two $(n-1)$ dimensional spheres, and let $f: R^{n} \rightarrow \bar{R}^{n}$ be a nonconstant quasimeromorphic map. Then there exists $r_{0}>0$ such that

$$
c v\left(\theta r, Y_{1}\right) \geq v\left(r, Y_{2}\right)
$$

for $r \geq r_{0}$.

We close this section by considering the question to what extent the inequalities in Theorems 4.1 and 4.8 hold for $\theta=1$. We obtain results similar to Ahlfors's well-known covering theorems, in other words, the inequalities hold outside a set of finite logarithmic measure. The proofs depend essentially on the following lemma.

4.14. L e m ma. Let $1<c<b, 0<c_{1}$, and let $\varphi$ and $\psi$ be two functions of the nonnegative real axis into itself such that $\psi$ is increasing and

$$
c \varphi(\theta r) \geq \psi(r)-c_{1}(\log \theta)^{1-n}
$$

for all $r>0$ and all $\theta>1$. Then

$$
b \varphi(r) \geq \psi(r)
$$

for all $r>0$ outside a set $E$ of finite logarithmic measure, i.e.

$$
\int_{E} \frac{d r}{r}<\infty .
$$

Proof. We shall apply a standard method, ef. [3, p. 38]. We may assume $\psi\left(r_{0}^{\prime}\right)>0$ for some $r_{0}^{\prime}>0$. We choose constants $M>1$ and $c_{2}>0$ such that $\left(1-c_{1} c_{2}^{n-1}\right) /(c M) \geq 1 / b$.

Set

$$
\beta(r)=\frac{c_{2} \psi(r)^{1 /(n-1)}}{p r}
$$

where $p>1$ is chosen so that for $r \geq r_{0}^{\prime}$

$$
\log \left(1+\frac{1}{r \beta(r)}\right) \geq \frac{1}{c_{2} \psi(r)^{1 /(n-1)}} .
$$

Let $F$ be the set of all $r>r_{0}^{\prime}$ such that 


$$
\psi(r+1 / \beta(r)) \leq M \psi(r)
$$

does not hold. Set $\theta_{r} r=r+1 / \beta(r)$. Let $r>r_{0}^{\prime}$ and $r \notin F$. Then

$$
\begin{aligned}
c \varphi\left(\theta_{r} r\right) & \geq \psi(r)-c_{1}\left(\log \left(1+\frac{1}{r \beta(r)}\right)\right)^{1-n} \\
& \geq\left(1-c_{1} c_{2}^{n-1}\right) \psi(r) \geq \frac{1}{M}\left(1-c_{1} c_{2}^{n-1}\right) \psi\left(\theta_{r} r\right) \\
& \geq \frac{c}{b} \psi\left(\theta_{r} r\right) .
\end{aligned}
$$

We now define a sequence $r_{0}^{\prime} \leq r_{1}<r_{1}^{\prime} \leq r_{2}<r_{2}^{\prime} \leq \ldots$ inductively as follows. Let $r_{k}$ be the infimum of all $r>r_{k-1}^{\prime}$ for which $r \in F$. If $r_{k}<\infty$, we then define $r_{k}^{\prime}=r_{k}+2 / \beta\left(r_{k}\right)$. Then $F$ is contained in the union $\mathrm{U}_{k \geq 1}\left[r_{k}, r_{k}^{\prime}\right]$. Let $F^{\prime}$ be the image of $F$ under the map $r \mapsto \theta_{r} r$. Suppose $\varrho>\theta_{r_{0}} r_{0}^{\prime}$ and $\varrho \notin F^{\prime}$. Since $\psi$ is increasing, it follows that there exists $r>r_{0}^{\prime}$ such that $\varrho=\theta_{r} r$. Then also $r \notin F$ and (4.16) holds. Thus by (4.17) it suffices to show that $F^{\prime}$ is contained in a set of finite logarithmic measure. To this end we observe that

$$
\psi\left(r_{k+1}\right) \geq \psi\left(r_{k}^{\prime}\right)=\psi\left(r_{k}+2 / \beta\left(r_{k}\right)\right) \geq M \psi\left(r_{k}\right) .
$$

The set $F^{\prime}$ is contained in the union

$$
E=\underset{k \geq 1}{\cup}\left[r_{k}, \varrho_{k}\right]
$$

where $\varrho_{k}=r_{k}^{\prime}+p r_{k}^{\prime} /\left(c_{2} \psi\left(r_{k}\right)^{1 /(n-1)}\right)$. Then we estimate

$$
\begin{aligned}
\int_{E} \frac{d r}{r} & \leq \sum_{k \geq 1} \int_{r_{k}}^{\varrho_{k}} \frac{d r}{r} \leq \sum_{k \geq 1}\left(r_{k}^{\prime}+\frac{p r_{k}^{\prime}}{c_{2} \psi\left(r_{k}\right)^{1 /(n-1)}}-r_{k}\right) / r_{k} \\
& =\sum_{k \geq 1} \frac{r_{k}^{\prime}-r_{k}}{r_{k}}\left(1+\frac{p}{c_{2} \psi\left(r_{k}\right)^{1 /(n-1)}}\right)+\sum_{k \geq 1} \frac{p}{c_{2} \psi\left(r_{k}\right)^{1 /(n-1)}} .
\end{aligned}
$$

Since $\left(r_{k}^{\prime}-r_{k}\right) / r_{k}=2 p /\left(c_{2} \psi\left(r_{k}\right)^{1 /(n-1)}\right)$, the sums are finite because of (4.18). The lemma is proved.

4.19. Th e o r e m. Let $c>1$, let $Y_{1}$ and $Y_{2}$ be two $(n-1)$-dimensional spheres in $\bar{R}^{n}$, and let $f: R^{n} \rightarrow \bar{R}^{n}$ be a nonconstant quasimeromorphic map. Then the inequalities

$$
\begin{gathered}
v\left(r, Y_{1}\right) / c \leq A(r) \leq c v\left(r, Y_{1}\right), \\
v\left(r, Y_{1}\right) \leq c v\left(r, Y_{2}\right)
\end{gathered}
$$

hold for all $r>0$ outside a set of finite logarithmic measure. 
Proof. For (4.20) we apply (4.9) and Lemma 4.14. The inequality (4.21) is a direct consequence of $(4.20)$.

4.22. Coroll a ry. If $Y_{1}, Y_{2}$, and $f$ are as in 4.19, then

$$
\begin{aligned}
& \liminf _{r \rightarrow \infty} \frac{A(r)}{v\left(r, Y_{1}\right)} \leq 1, \\
& \liminf _{r \rightarrow \infty} \frac{v\left(r, Y_{1}\right)}{A(r)} \leq 1, \\
& \liminf _{r \rightarrow \infty} \frac{v\left(r, Y_{1}\right)}{v\left(r, Y_{2}\right)} \leq 1 .
\end{aligned}
$$

Proof. One applies 4.19 to a sequence $c_{1}, c_{2}, \ldots$ of constants $c$ tending to 1 .

\section{Relations between the counting function and the spherical average}

In this section we shall study inequalities of the type $n(r, \alpha) \leq c A(\theta r)$ where $c$ and $\theta$ are constants greater than 1. For a nonconstant meromorphic function $f: R^{2} \rightarrow \bar{R}^{2}$ it follows trivially from the AhlforsShimizu form of Nevanlinna's first main theorem that

$$
\liminf _{r \rightarrow \infty} \frac{n(r, a)}{A(r)} \leq 1
$$

if $a \in \bar{R}^{2}$. For quasimeromorphic maps $f: R^{n} \rightarrow \bar{R}^{n}$ the left hand side of (5.1) may exceed 1 , but it is not known whether it must be finite and hence whether it has an upper bound which does not depend on $a$. However, one can prove (Theorem 5.16) that for each $c>1$ there exists $\theta>1$ such that

$$
\liminf _{r \rightarrow \infty} \frac{n(r, a)}{A(\theta r)} \leq c .
$$

It is interesting not only from the point of view of quasimeromorphic maps but also of meromorphic functions in the plane to study the validity of inequalities of type

$$
n(r, a) \leq c A(\theta r) \quad \text { if } r \geq r_{0} .
$$

That (5.3) need not hold in the general case even for meromorphic functions no matter how the constants $c$ and $\theta$ are chosen follows from a modification of an example of a meromorphic function given by Toppila [10, Theorem 
4]. However, at the presence of an asymptotic value $a_{0}$, for each $c>1$ there exists $\theta>1$ such that, whenever $a$ lies in a compact set not containing $a_{0},(5.3)$ holds for a fixed $r_{0}$ (Theorem 5.11). For the general case one can show that an inequality $n(r, a) \leq c A\left(r^{\alpha}\right)$ holds where $\alpha>1$ (Theorem 5.22). According to [10, Theorem 4] a power of $r$ is the best one can hope for.

The proof of the following lemma contains the basic idea to obtain the various results in this section.

5.4. L e m m a. Let $f: R^{n} \rightarrow \bar{R}^{n}$ be a nonconstant quasimeromorphic $\operatorname{map}$, let $1<c^{\prime}, 1<u<v, t>0$, and $r>0$. Set

$$
\begin{aligned}
H(r, t) & =\left\{\lambda \in[r, u r] \mid S(\lambda) \cap f^{-1} \mathbf{C} B(t) \neq \varnothing\right\}, \\
\varphi(r, t) & =\int_{H(r, t)} \frac{d \lambda}{\lambda} .
\end{aligned}
$$

Then

$$
v(v r, t) \geq\left(\frac{1}{c^{\prime}}-\frac{\omega_{n-1} K_{o}(f) K_{I}(f)}{\left(c^{\prime}-1\right) c_{n} \varphi(r, t)(\log (v / u))^{n-1}}\right) n(r, 0),
$$

where $c_{n}>0$ is the constant in $[11,(10.11)]$ depending only on $n$.

Proof. Let $0<s_{0}<t$ be such that

$$
\left(\log \frac{t}{s_{0}}\right)^{n-1}>\frac{K_{O}(f) \omega_{n-1} n(r, 0)}{c_{n} \varphi(r, t)} .
$$

We claim that there exists $s^{\prime} \in\left[s_{0}, t\right]$ such that

$$
v\left(u r, s^{\prime}\right) \geq n(r, 0)
$$

holds. We choose $s \in] s_{0}, t[$ such that

$$
\left(\log \frac{t}{s}\right)^{n-1}>\frac{K_{o}(f) \omega_{n-1} n(r, 0)}{c_{n} \varphi(r, t)} .
$$

We consider separately two cases:

Case 1. For every $x \in f^{-1}(0) \cap \bar{B}(r)$ the $x$-component of $f^{-1} B(s)$ is contained in $B(u r)$.

Case 2. There exists $x \in f^{-1}(0) \cap \bar{B}(r)$ such that the $x$-component of $f^{-1} B(s)$ intersects $\mathbf{C} B(u r)$.

Assume Case 1 and let $D$ be an $x$-component of $f^{-1} B(s)$ with $x \in f^{-1}(0) \cap \bar{B}(r)$. Then $D$ is a normal domain $[4,2.5]$ and hence

$$
\sum_{z \in f^{-1}\left(s_{0} y\right) \cap D} i(z, f)=\sum_{x \in f^{-1}(0) \cap D} i(x, f)
$$

for all $y \in S$. Summing over all such $D$ gives 


$$
n\left(u r, s_{0} y\right) \geq \sum_{D} \sum_{z \in f^{-1}\left(s_{0} y\right) \cap D} i(z, f)=\sum_{D} \sum_{x \in f^{-1}(0) \cap D} i(x, f) \geq n(r, 0)
$$

for all $y \in S$. Hence (5.7) is satisfied with $s^{\prime}=s_{0}$.

Assume Case 2 and suppose

$$
v\left(u r, s^{\prime}\right)<n(r, 0)
$$

for all $s^{\prime} \in\left[s_{0}, t\right]$. Let $\Gamma$ be the family of paths joining $f^{-1} \mathbf{C} B(t)$ and $f^{-1} B(s)$ in $B(u r)$. Let $D$ be an $x$-component of $f^{-1} B(s)$ which intersects C $B(u r)$ and where $x \in \bar{B}(r)$. Since every $S(\lambda)$ intersects $D$ for $r<\lambda<$ $u r$, we obtain by integrating $[11,(10.10)]$ the estimate

$$
M(\Gamma) \geq c_{n} \int_{H(r, t)} \frac{d \lambda}{\lambda}=c_{n} \varphi(r, t) .
$$

We define a non-negative function $\varrho$ of $R^{n}$ by

$$
\begin{aligned}
& \varrho(z)=\frac{1}{|z| \log \frac{t}{s}} \text { if } s \leq|z| \leq t, \\
& \varrho(z)=0 \text { elsewhere. }
\end{aligned}
$$

Then $\varrho \in F(f T)$. We now use $(5.9),[4,8.3,8.4]$, and the proof of $[4,3.2]$ and obtain

$$
\begin{aligned}
M(\Gamma) & \leq K_{O}(f) \int_{R^{n}} \varrho(z)^{n} n(u r, z) d m(z) \\
& =K_{O}(f) \int_{s}^{t}\left(\int_{S} \frac{n(u r, \tau y)}{(\log (t / s))^{n} \tau} d \mathscr{H}^{n-1}(y)\right) d \tau \\
& =K_{O}(f) \int_{s}^{t} \frac{\omega_{n-1} v(u r, \tau)}{(\log (t / s))^{n} \tau} d \tau \leq \frac{K_{O}(f) \omega_{n-1} n(r, 0)}{(\log (t / s))^{n-1}} .
\end{aligned}
$$

Combined with (5.8) and (5.10) this gives a contradiction. Hence also in this case (5.7) holds for some $s^{\prime} \in\left[s_{0}, t\right]$.

Now we use Theorem 4.1 and (5.7) to conclude

$$
\begin{aligned}
v(v r, t) & \geq \frac{1}{c^{\prime}} v\left(u r, s^{\prime}\right)-\frac{K_{I}(f)\left(\log \left(t / s^{\prime}\right)\right)^{n-1}}{\left(c^{\prime}-1\right)(\log (v / u))^{n-1}} \\
& \geq \frac{1}{c^{\prime}} n(r, 0)-\frac{K_{I}(f)\left(\log \left(t / s_{0}\right)\right)^{n-1}}{\left(c^{\prime}-1\right)(\log (v / u))^{n-1}} .
\end{aligned}
$$


Since this holds for all choices $s_{0}$ in (5.6), we obtain (5.5). The lemma is proved.

5.11. T h e o r e m. Let $f: R^{n} \rightarrow \bar{R}^{n}$ be a nonconstant quasimeromorphic map and let $f$ have an asymptotic value $a_{0}$. Then for each $c>1$ there exists $\theta>1$, depending only on $n, c$, and the dilatation $K(f)$, such that whenever $E \subset \overline{R^{n}} \backslash\left\{a_{0}\right\}$ is a compact set, there exists $r_{0}>0$ such that

$$
\sup _{a \in E} n(r, a) \leq c A(\theta r)
$$

for $r \geq r_{0}$.

Proof. Let $c=c^{\prime 3}>1$. Since $a_{0}$ is an asymptotic value, there exists a path $\alpha:\left[0,1\left[\rightarrow R^{n}\right.\right.$ such that $\alpha(\tau) \rightarrow \infty$ and $f(\alpha(\tau)) \rightarrow a_{0}$ as $\tau \rightarrow 1$. Let $\delta$ be the spherical chordal distance between $a_{0}$ and $E$. To prove (5.12) let $a \in E$. By performing a Möbius transformation which preserves spherical distances we may assume $a=0$. Let $B(t)$ be a ball whose spherical radius is $\delta / 2$. There exists $\tau_{1} \in\left[0,1\left[\right.\right.$ such that $f(\alpha(\tau)) \in \bar{R}^{n} \backslash$ $B(t)$ if $\tau_{1} \leq \tau<1$. Let $r \geq\left|\alpha\left(\tau_{1}\right)\right|, 1<u<v$, and set $\alpha_{1}=\alpha \mid\left[\tau_{1}, 1[\right.$.

Since $\alpha_{1}$ is contained in $f^{-1} \mathbf{C} B(t)$, every $S(\lambda)$ intersects $f^{-1} \mathbf{C} B(t)$ for $\lambda \geq r$. Then in Lemma $5.4 H(r, t)=[r, u r]$ and hence $\varphi(r, t)=\log u$. We get then by 5.4

$$
v(v r, t) \geq\left(\frac{1}{c^{\prime}}-\frac{\omega_{n-1} K(f)^{2}}{\left(c^{\prime}-1\right) c_{n} \log u(\log (v / u))^{n-1}}\right) n(r, 0) .
$$

Now we choose $v=u^{2}$ and $u$ so that the factor in front of $n(r, 0)$ in (5.13) equals $c^{\prime} / c$. Let then $\theta=2 v$. By Theorem 4.8 there exists $r_{0} \geq$ $\left|\alpha\left(\tau_{1}\right)\right|$ such that

$$
A(\theta r) \geq \frac{1}{c^{\prime}} v(v r, t)
$$

for $r \geq r_{0}$. Then $n(r, 0) \leq c A(\theta r)$ for $r \geq r_{0}$, where $\theta$ depends only on $n, c$, and $K(f)$. It follows from (4.9) that $r_{0}$ can be chosen to depend on $\delta$ but not on the particular point $a \in E$ chosen. The theorem is proved.

5.14. Corollary. Let $f: R^{n} \rightarrow \bar{R}^{n}$ be a quasimeromorphic map which has at least two asymptotic values. Then for each $c>1$ there exists $\theta>1$, depending only on $n, c$, and $K(f)$, such that

$$
\sup _{a \in \bar{R}^{n}} n(r, a) \leq c A(\theta r)
$$

if $r \geq r_{0}$ for some $r_{0}$. 
5.15. R e mark. Toppila has shown by examples of meromorphic functions in plane [10, Theorem 4, Theorem 6] that the assumptions of the existence of the asymptotic values cannot be left out in 5.11 and 5.14 . He has also shown that $\theta$ cannot be chosen arbitrary near 1 even in the meromorphic case [10, Theorem 5].

For the general case we have the following result.

5.16. Th e o r e m. Let $f: R^{n} \rightarrow \bar{R}^{n}$ be a nonconstant quasimeromorphic map. Then for each $c>1$ there exists $\theta>1$, depending only on $n, c$, and $K(f)$, such that for every $a \in \bar{R}^{n}$

$$
\liminf _{r \rightarrow \infty} \frac{n(r, a)}{A(\theta r)} \leq c .
$$

Proof. Let $c=c^{4}>1$ and $a \in \bar{R}^{n}$. We may assume $a=0$. We are going to show that for a given $r_{0}>0$ an inequality $n\left(r_{1}, a\right) \leq c A\left(\theta r_{1}\right)$ is true for some $r_{1} \geq r_{0}$. We may assume $n\left(r_{0}, 0\right)>0$ and by 4.8 that $r_{0}$ is so large that for $\varrho \geq r_{0}$

$$
A(2 \varrho) \geq \frac{1}{c^{\prime}} v(\varrho, 1) .
$$

In order to apply Lemma 5.4 we define first $u>1$ by the formula

$$
\frac{1}{c^{\prime 2}}=\frac{1}{c^{\prime}}-\frac{3 \omega_{n-1} K(f)^{2}}{\left(c^{\prime}-1\right) c_{n}(\log u)^{n}} .
$$

With the notation of 5.4 let

$$
\psi(t)=\sup _{r \geq r_{0}} \varphi(r, t) .
$$

Suppose first $\psi(1) \geq(\log u) / 2$. Let $r_{1} \geq r_{0}$ be such that $\varphi\left(r_{1}, 1\right) \geq$ $(\log u) / 3$. We apply 5.4 with $v=u^{2}$ and get

$$
v\left(v r_{1}, 1\right) \geq\left(\frac{1}{c^{\prime}}-\frac{3 \omega_{n-1} K(f)^{2}}{\left(c^{\prime}-1\right) c_{n}(\log u)^{n}}\right) n\left(r_{1}, 0\right) \text {. }
$$

Putting $\varrho=v r_{1}$ and $\theta=2 v$ (5.17) and (5.18) give then $n\left(r_{1}, 0\right) \leq$ $c^{\prime 3} A\left(\theta r_{1}\right)<c A\left(\theta r_{1}\right)$.

Suppose next that $\psi(1)<(\log u) / 2$. We observe that for every $r>0 \quad t \mapsto \varphi(r, t)$ is decreasing and $\varphi(r, t) \rightarrow \log u$ as $t \rightarrow 0$, hence

$$
t_{r_{0}}=\inf \{t \leq 1 \mid \psi(t) \leq(\log u) / 2\}
$$

is positive. We may assume $t_{r_{0}}<1$. Assume $0<\delta<\min \left\{t_{r_{0}}, 1-t_{r_{0}}\right\}$ and set $t_{1}=t_{r_{0}}-\delta, t_{1}^{\prime}=t_{r_{0}}+\delta$. Then $\psi\left(t_{1}\right)>(\log u) / 2$. Let $r_{1} \geq r_{0}$ 
be such that $\varphi\left(r_{1}, t_{1}\right) \geq(\log u) / 2$. Then Lemma 5.4 gives for $v=u^{2}$ the estimate

$$
\begin{aligned}
v\left(v r_{1}, t_{1}\right) & \geq\left(\frac{1}{c^{\prime}}-\frac{2 \omega_{n-1} K(f)^{2}}{\left(c^{\prime}-1\right) c_{n}(\log u)^{n}}\right) n\left(r_{1}, 0\right) \\
& >\frac{1}{c^{\prime 2}} n\left(r_{1}, 0\right) .
\end{aligned}
$$

Since $n\left(r_{1}, 0\right) \geq n\left(r_{0}, 0\right)>0$, we may by Theorem 4.1 choose $\delta$ originally so small that

$$
v\left(w r_{1}, t_{1}^{\prime}\right) \geq \frac{1}{c^{\prime}} v\left(v r_{1}, t_{1}\right)
$$

if $w \geq 2 v$. Let $t_{\gamma_{0}}<t<t_{1}^{\prime}$. Since $\psi(t) \leq(\log u) / 2$, there exists $\varrho \in\left[2 v r_{1}, 2 u v r_{1}\right]$ such that $S(\varrho) \cap f^{-1} \mathbf{C} B(t)=\varnothing$. Then every component of $f^{-1} \mathbf{C} \bar{B}(t)$ which meets $\bar{B}(\varrho)$ is a normal domain contained in $B(\varrho)$ $[4,2.5]$. Hence $n(y, \varrho)=n(z, \varrho)$ for all $y, z \in \mathbf{C} \bar{B}(t)$. It follows that

$$
v\left(\varrho, t_{1}^{\prime}\right)=v(\varrho, 1) .
$$

We choose $w=\varrho / r_{1}$ and $\theta=4 u^{3} \geq 2 w$. Then (5.17) and (5.19)(5.21) give the desired inequality $n\left(r_{1}, 0\right) \leq c A\left(\theta r_{1}\right)$. The theorem is proved.

We obtain a lim sup result also for the general case if we replace $\theta r$ by a power of $r$, uniformly with respect to the point $a$ :

5.22. Th e o r e m. Let $f: R^{n} \rightarrow \bar{R}^{n}$ be a nonconstant quasimeromorphic map. Then for each $c>1$ there exists $\alpha>1$, depending only on $n, c$, and $K(f)$, such that

$$
\limsup _{r \rightarrow \infty} \frac{n(r)}{A\left(r^{\alpha}\right)} \leq c
$$

where $n(r)=\sup _{a \in \bar{R}^{n}} n(r, a)$.

Proof. The idea of the proof is to find a substitute for the estimate (5.10) of $M(\Gamma)$ in the proof of Lemma 5.4. Let the balls $B_{1}=B\left(s_{1}\right)$ and $B_{2}=\mathbf{C} \bar{B}\left(s_{2}\right)$ have both spherical chordal radius $1 / 4$. Then for each point $a \in \bar{R}^{n}$ the spherical chordal distance $q\left(a, B_{i}\right)$ is greater than $1 / 4$ for at least one $i$. There exist $\varrho_{0}>0$ and balls $U_{1}, U_{2} \subset B\left(\varrho_{0}\right)$ such that $f U_{i} \subset B_{i}$, $i=1,2$. Let now $a \in \bar{R}^{n}$ and suppose $q\left(a, B_{1}\right)>1 / 4$. Let $T$ be a Möbius transformation preserving spherical distances such that $T(a)=0$. Let $B(t)$ have spherical chordal radius $1 / 4$. Then $T\left(f U_{1}\right) \cap B(t)=\varnothing$. Set $g=T \circ f$. 
We shall use almost the same argument as in the proof of Lemma 5.4 for the map $g$ and in a situation where $c^{\prime 3}=c, r>\varrho_{0}, u=2$, and $u<v$. If $\Gamma_{1}$ is a family of paths joining $U_{1}$ and a continuum which connects $S(r)$ and $S(2 r)$ in $B(2 r)$, then from an $n$-dimensional version of $[1$, Theorem 4$]$ it follows that

$$
M\left(\Gamma_{1}\right) \geq d_{n}(\log r)^{1-n}
$$

if $r \geq r_{0}$ for some $r_{0}>\varrho_{0}$ where $d_{n}>0$ is a constant which depends only on $n$. In the proof of 5.4 we now replace in (5.6) and $(5.8) c_{n} \varphi(r, t)$ by the right hand side of (5.23). Consider the Case 2 in the proof 5.4. If $\Gamma$ has the corresponding definition, i.e. it is the family of paths joining $g^{-1} \mathbf{C} B(t)$ and $g^{-1} B(s)$ in $B(2 r)$, then $M(\Gamma) \geq M\left(\Gamma_{1}\right)$. Then we obtain for $r \geq r_{0}$

$$
v_{g}(v r, t) \geq\left(\frac{1}{c^{\prime}}-\frac{\omega_{n-1} K(f)^{2}(\log r)^{n-1}}{\left(c^{\prime}-1\right) d_{n}(\log (v / 2))^{n-1}}\right) n_{g}(r, 0)
$$

where we now have used a subscript $g$ to indicate the map. By Theorem 4.8 there exists $r_{1} \geq \max \left(r_{0}, 2\right)$ such that

$$
A_{f}(2 v r)=A_{g}(2 v r) \geq \frac{1}{c^{\prime}} v_{g}(v r, t)
$$

if $r \geq r_{1}$ and where $r_{1}$ does not depend on the point $a$. We choose $v$ so that the factor in front of $n_{g}(r, 0)$ in $(5.24)$ is $1 / c^{\prime 2}$. Then $2 v r \leq r^{\alpha}$ for $r \geq r_{1}$ where $\alpha>1$ is a constant, depending only on $n, c$, and $K(f)$, and (5.24) and (5.25) give the desired result $n_{f}(r, a) \leq c A_{f}\left(r^{\alpha}\right)$ for $r \geq r_{1}$. The proof for $q\left(a, B_{2}\right)>1 / 4$ is similar.

\section{References}

[1] Gehring, F. W.: Symmetrization of rings in space. - Trans. Amer. Math. Soc. 101, 1961, 499-519.

[2] af HÄLISTRÖM, G.: Wertverteilungssätze meromorpher Funktionen. - Acta Acad. Åbo. Ser. B 21, N:o 9, 1958, 1-23.

[3] Hayman, W. K.: Meromorphic functions. - Clarendon Press, Oxford, 1964.

[4] Martio, O., S. RICKMAN, and J. VÄISÄLÄ: Definitions for quasiregular mappings. - Ann. Acad. Sci. Fenn. Ser. A I 448, 1969, 1-40.

[5] -»- -»- -»- Distortion and singularities of quasiregular mappings. - Ibid. 465, 1970, 1-13.

[6] -»- -»- -"- Topological and metric properties of quasiregular mappings. - Ibid. $488,1971,1-31$.

[7] POLЕскї, Е. А. (Е. А. Полецкий): Метод модулей для негомеоморфных квазиконформных отображений. - Mat. Sb. 83, 1970, 261-272. 
[8] Rickman, S.: Path lifting for discrete open mappings. - Duke Math. J. 40, 1973, 187-191.

[9] -》- A quasimeromorphic mapping with given deficiencies in dimension three. - To appear in Symposia Math.

[10] Toppila, S.: On the counting function for the $a$-values of a meromorphic function. - Ann. Acad. Sci. Fenn. Ser. A I 2, 1976,

[11] VÄIsÄL $\ddot{A}, J .:$ Lectures on $n$-dimensional quasiconformal mappings. - Lecture Notes in Mathematics 229, Springer-Verlag, Berlin-HeidelbergNew York, 1971.

[12] -»- Modulus and capacity inequalities for quasiregular mappings. - Ann. Acad. Sci. Fenn. Ser. A I 509, 1972, 1-14.

[13] ZiEMER, W. P.: Extremal length and p-capacity. - Michigan Math. J. 16, $1969,43-51$.

University of Helsinki

Department of Mathematics

SF-00100 Helsinki 10

Finland

Received 7 October 1975 